\title{
FIBRÉS HOLOMORPHES SUR UN TORE COMPLEXE
}

\author{
YOZÔ MATSUSHIMA
}

\section{Introduction}

Le problème de classifier les fibrés vectoriels holomorphes sur une variété complexe $M$ a été résolu récemment dans les deux cas suivants: 1) $M$ est la sphère de Riemann (Grothendieck [4]),2) $M$ est une courbe elliptique (Atiyah [2]). Mais si la dimension complexe de $M$ est $>1$, nous ne connaissons presque rien de ce problème, sauf le cas où la dimension complexe de fibre est égale à 1 .

Nous nous proposons ici d'étudier quelques propriétés des fibrés vectoriels holomorphes sur un tore complexe qui possèdent une connexion holomorphe. Nos résultats nous conduirons à la classification de tels fibrés dans le cas où la dimension complexe de fibre est égale à 2 (Théorème 4): II y a une correspondance biunivoque bien déterminée entre les classes de tels fibrés indécomposables et la variété $T^{\prime n} \times P^{n-1}$, où $T^{\prime n}$ désigne la variété de Picard de la base (la base étant un tore complexe de dimension complexe $n$ ) et où $P^{n-1}$ désigne l'espace projectif complexe de dimension complexe $n-1$. Notons ici que la condition qu'un fibré vectoriel holomorphe possède une connexion holomorphe est équivalente, 1) si la base est de dimension complexe 1, à la condition que ce fibré se définit par une représentation du groupe fondamental de la base et, 2) si la base est une variété kaehlerienne compacte et si la dimension de fibré est égale à 1 , à la condition que sa classe de Chern est nulle.

Notre méthode est essentiellement différente de celle de Atiyah [2]. Il y a de difficultés d'étendre la méthode de Atiyah dans le cas où la base est un tore complexe de dimension complexe $>1$. La base de notre étude est le théorème 1 qui dit: Un fibré principal holomorphe sur un tore complexe pos-

Received August 8, 19,58. 
sède une connexion holomorphe si et seulement s'il est homogène. Ici on dit qu'un fibré principal holomorphe est homogène si son groupe d'automorphismes (qui est de Lie complexe d'après Morimoto [7]) est transitif sur les fibres. Ce théorème sera utilisé pour traiter les questions relatives à la décomposition en facteurs indécomposables (Théorème 2 , Corollaire) et à la réduction du groupe structural (Théorème 3 ). Comme un corollaire du théorème 3 nous verrons que les fibrés vectoriels holomorphes sur un tore complexe admettant une connexion holomorphe sont topologiquement triviaux. Le théorème 4 cité au-dessus résulte du théorème 3.

Nous notons que Murakami [8] a obtenu, par une méthode très voisine de la nôtre, des résultats intéressants sur les fibrés holomorphes sur un tore complexe, de groupe structural abélien, qui possèdent une connexion holomorphe.

J'ai tiré profit de plusieurs discussions avec A. Morimoto sur les questions traitées ici, je tiens à l'en remercier.

\section{Notations. Rappel des résultats.}

A. Fibrés holomorphes et connexions holomorphes. Soit $P(M, U, \pi)$ un fibré principal holomorphe de base $M$, de groupe structural $U$ et de projection $\pi$. Soit $\Gamma$ une connexion différentiable sur le fibré $P(M, U, \pi){ }^{1)} \quad$ Désignons par $T_{x}$ l'espace tangent de $P$ au point $x$. Soit $Q_{x}$ le sous-espace horizontal de $T_{x}$ défini par la connexion $\Gamma$. Soit $\omega$ la forme de connexion de $I$. On dit que la connexion $\Gamma$ est de type $(1,0)$ (resp. holomorphe) si la forme $\omega$ est de type $(1,0)$ (resp. holomorphe) par rapport à la structure complexe de $P$. Soit $I_{P}$ le tenseur sur $P$ définissant la structure complexe de $P$. On voit alors que la connexion $\Gamma$ est de type $(1,0)$ si et seulement si l'on a $I_{P} \cdot Q_{x} \subset Q_{x}$ pour tout $x \in P$. Chaqu'une des conditions suivantes est nécessaire et suffisante pour qu'une connexion $\Gamma$ de type $(1,0)$ soit holomorphe:

1) Pour tout champ de vecteurs holomorphe $X$ défini dans un ouvert de $P$ la composante horizontale $h X$ de $X$ relative à $\Gamma$ est holomorphe.

1) Pour la notion de connexion dans un fibré nous suivons Nomizu [9]. Pour la théorie générale de la connexion holomorphe, voir également Atiyah [1]. Dans tout ce qui suit les variétés considérées seront supposées vérifiant le second axiome de dénombrabilité. Pour un groupe de Lie $G$, ceci signifie que le nombre des composantes connexes de $G$ est dénombrable. 
2) Pour tout champ de vecteurs holomorphe $X$ défini dans un ouvert de $M$ le relèvement $X^{*}$ de $X$ relatif à $l$ est holomorphe.

Un homomorphisme d'un fibré holomorphe $P^{\prime}\left(M, U^{\prime}, \pi^{\prime}\right)$ dans un fibré holomorphe $P(\dot{M}, U, \pi)$ est défini par une application holomorphe $\alpha$ de $P^{\prime}$ lans $P$ et par un homomorphisme holomorphe $\alpha$ de $U^{\prime}$ dans $U$ tels que $\pi^{\prime}\left(x^{\prime}\right)$ $=\pi\left(\alpha\left(x^{\prime}\right)\right), \alpha\left(x^{\prime} \cdot a^{\prime}\right)=\alpha\left(x^{\prime}\right) \cdot \alpha\left(a^{\prime}\right)$ pour tout $x^{\prime} \in P$ et $a^{\prime} \in U^{\prime}$.

Soit maintenant $P^{\prime}\left(M, U^{\prime}, \pi^{\prime}\right)$ un fibré holomorphe et soit $\alpha$ un homomorphisme holomorphe de $U^{\prime}$ dans un groupe de Lie complexe $U$. Soit $P(M$, $U, \pi)$ le fibre principal holomorphe associé à $P^{\prime}\left(M, U^{\prime}, \pi^{\prime}\right)$ par l'homorphisme $\alpha ; P$ est l'espace quotient de $P^{\prime} \times U$ par la relation d'équivalence, $\left(x^{\prime}, b\right) \sim$ $\left(x^{\prime} a^{\prime}, \alpha\left(a^{\prime}\right)^{-1} \cdot b\right) \quad\left(x^{\prime} \in P^{\prime}, b \in U, a^{\prime} \in U^{\prime}\right)$. On voit qu'un fibré holomorphe $P(M, U, \pi)$ est associé à $P^{\prime}\left(M, U^{\prime}, \pi^{\prime}\right)$ par un homomorphisme holomorphe de $U^{\prime}$ dans $U$ si et seulement s'il existe un homomorphisme de $P^{\prime}\left(M, U^{\prime}, r^{\prime}\right)$ dans $P(M, U, \pi)$.

Soit $\alpha$ un homomorphisme d'un fibré holomorphe $P^{\prime}\left(M, U^{\prime}, \pi^{\prime}\right)$ dans un fibré holomorphe $P(M, U, \pi)$ et soit $\Gamma^{\prime}$ un connexion différentiable sur $P^{\prime}$. Alors $\alpha$ et $\Gamma^{\prime}$ définissent une connexion $\alpha \cdot \Gamma^{\prime}$ sur $P$ ([9], p 36). On vérifie sans difficulté que si $\Gamma^{\prime}$ est holomorphe, $\alpha \cdot \Gamma^{\prime}$ l'est aussi. Par suite si le fibré holomorphe $P^{\prime}\left(M, U^{\prime}, \pi^{\prime}\right)$ possède une connexion holomorphe, tout fibré holomorphe associé à $P^{\prime}\left(M, U^{\prime}, \pi^{\prime}\right)$ en possède une.

Notations. Dans tout ce qui suit nous utiliserons les notations suivantes. $C^{*}$ : Le groupe multiplicatif des nombres complexes $\neq 0, C$ : Le corps des nombres complexes, $R$ : Le corps des nombres réels.

Remarque. Pour un fibré principal holomorphe $P\left(M, C^{*}, \pi\right)$ sur une variété kählérienne compacte $M$, de groupe structural $C^{*}$ les trois conditions suivantes sont équivalentes. 1) $P$ possède une connexion holomorphe, 2) la classe de Chern de $P$ est nulle, 3) $P$ est topologiquement trivial. Par suite les classes d'équivalence de fibrés sur $M$, de groupe structural $C^{*}$, qui contiennent des fibrés possédant une connexion holomorphe constituent la variété de Picard de $M$ (cf. Kodaira and Spencer [6]).

2) Les notions de la composante horizontale et du relèvement d'un champ de vecteurs ont été définies dans [9] seulement pour les champs de vecteurs réels. On étend ces notions pour les champs de vecteurs complexes par linéarité. 
B. Automorphismes d'un fibré holomorphe. Soit $P(M, U, \pi)$ un fibré holomorphe. Un automorphisme $f$ de $P(M, U, \pi)$ est un automorphisme de la variété complexe $P$ tel que $f(x \cdot a)=f(x) \cdot a$ pour tout $x \in P$ et $a \in U$. Soit $F(P)$ le groupe d'automorphismes de $P(M, U, \pi)$. Si la base $M$ est compacte, $F(P)$ se munit d'une structure d'un groupe de Lie complexe telle que l'application canonique de $F(P) \times P$ dans $P$ définie par $(f, x) \rightarrow f(x)$ soit holomorphe. ${ }^{3)}$ L'algèbre de Lie $\mathfrak{f}(P)$ de $F(P)$ s'identifie avec l'algèbre de tous les champs de vecteurs holomorphes $X$ sur $P$ tels que $R_{a}^{\prime} \cdot X=X$ pour tout $a \in U$, où $R_{a}^{\prime}$ désigne la différentielle de la translation à droite $R_{a}$ de $P$ par $a \in U$ (Morimoto [7]). La structure complexe de l'algèbre $f(P)$ est définie en posant $\sqrt{-1} X=I_{P} \cdot X$ pour tout $X \in \mathfrak{f}(P)$, où $I_{P}$ désigne le tenseur définissant la structure complexe de $P$. Soit $A(M)$ le groupe d'automorphismes de la variété complexe et compacte $M . \quad A(M)$ se munit d'une structure d'un groupe de Lie complexe et l'algèbre de Lie $a(M)$ de $A(M)$ s'identifie avec l'algèbre de tous les champs de vecteurs holomorphes sur $M$. Il existe un homomorphisme holomorphe $\pi$ de $F(P)$ dans $A(P)$ tel que $(\pi f)(\pi(x))=\pi(f(x))$ pour tout $x \in P$. Soit $\pi^{\prime}$ $l^{\prime}$ homomorphisme induit de $f(P)$ dans $a(M)$. Alors pour tout $X \in \mathfrak{f}(P), \pi^{\prime}(X)$ est égal à la projection sur $M$ du champ de vecteurs $X \in \mathfrak{f}(P)$. (Remarquons que tout champ de vecteurs $X$ dans $\mathfrak{f}(P)$ est projetable sur $M$, car $R_{a}^{\prime} \cdot X=X$ pour tout $a \in U$.)

Considérons maintenant le cas $U=G L(m, C)$. Soit $E$ le fibré vectoriel holomorphe de fibre $C^{m}$ associé au fibré holomorphe $P(M, G L(m, C), \pi)$. Pour tout $u \in M$ désignons par $E_{u}$ la fibre de $E$ au-dessus de $u$. Un automorphisme $f$ du fibré vectoriel $E$ est un automorphisme de la variété complexe $E$ satisfaisant aux conditions suivantes.

a) Il existe un automorphisme $\bar{f}$ de la base $M$ (qui sera appelé la projection de $f)$ tel que $f\left(E_{u}\right)=E_{\bar{f}}^{-}(u)$ pour tout $u \in M$.

b) La restriction de $f$ à la fibre $E_{u}$ est une application linéaire de $E_{u}$ sur $E_{\tilde{f}(u)}$ pour tout $u \in M$.

Soit $F(E)$ le groupe d'automorphismes de $E$. Nous allons montrer que l'on peut identifier $F(E)$ avec $F(P)$. L'espace $E$ est le quotient de $P \times C^{m}$. par la relation d'équivalence $(x, \xi) \sim\left(x \cdot a, a^{-1} \cdot \xi\right)\left(x \in P, \xi \in C^{m}, a \in G L(m, C)\right)$. Soit

3) Le groupe $F(P)$ satisfait au second axiome de dénombrabilité, 
$\rho$ la projection canonique de $P \dot{x} C^{m}$ sur $E$. Soit $f \in F(P)$. $f$ étant un automorphisme du fibré $P$, on peut définir une application $f^{\prime}$ de $E$ sur lui-même par $f^{\prime}(\rho(x, \xi))=\rho(f(x), \xi)\left(x \in P, \xi \in C^{m}\right)$. On vérifie facilement que $f^{\prime}$ est un automorphisme du fibré vectoriel $E$ et que la projection de $f^{\prime}$ est égale à la projection $\bar{f}$ de $f$. De plus, l'application $\theta: F(P) \rightarrow F(E)$ définie par $\theta(f)=f^{\prime}$ est un isomorphisme du groupe $F(P)$ dans le groupe $F(E)$. Soit, réciproquement, $f^{\prime}$ un automorphisme du fibré vectoriel $E$. Pour tout $x \in P$ fixé, il existe $y \in P$ tel que $f^{\prime}(\rho(x, \xi))=\rho(y, \hat{\xi})$ pour tout $\xi \in C^{m}$. Posons $f(x)=y$. On voit alors que $f$ est un automorphisme de $P(M, G L(m, C), \pi)$ et que $\theta(f)=f^{\prime}$. Par conséquent $\theta$ est un isomorphisme de $F(P)$ sur $F(E)$.

C. Tores complexes. Nous désignons dans tout ce qui suit par $T^{n}$ un tore complexe de dimension complexe $n$. On identifie $T^{n}$ avec un sous-groupe du groupe d'automorphismes $A\left(T^{n}\right)$ de la variété complexe $T^{n}$. On sait que $T^{n}$ coïncide avec la composante connexe de l'élément neutre de $A\left(T^{n}\right)$.

Soit $T^{n}=A / \Pi$, où $A$ est un espace vectoriel complexe de dimension complexe $n$ et où II est un sous-groupe discret de $A$ de rang $2 n$. On peut considérer $A$ comme un espace vectoriel réel de dimension réel $2 n$ que l'on désigne par $A_{0}$. La structure complexe de $A$ est définie par un automorphisme $J$ de $A_{0}$ tel que $J^{2}=-I$, où $I$ désigne l'automorphisme identique de $A_{0}$. Soit maintenant $H_{1}\left(T^{n}, Z\right.$ ) (resp. $H_{1}\left(T^{n}, R\right)$ ) le groupe d'homologie de dimension 1 à coefficients entiers (resp. à coefficients réels). Il existe un isomorphisme canonique $\varphi$ de $\Pi$ sur $H_{1}\left(T^{n}, Z\right)$. $\varphi$ est défini comme suit: soit $a \in \Pi$ et soit $C_{a}$ une courbe joignant 0 et $a, 0$ étant l'origine de $A_{0}$. Soit $C_{a}^{\prime}$ la courbe fermée de $T^{n}$ projection de $C_{a}$. Alors $\varphi(a)$ est la classe d'homologie de $T^{n}$ contenant le cycle $C_{c}^{\prime}$. Les espaces vectoriels $A_{0}$ et $H_{1}\left(T^{n}, R\right)$ sont engendrés sur $R$ respectivement par $I I$ et par $H_{1}\left(T^{n}, Z\right)$. On peut donc prolonger $\varphi$ à un isomorphisme $\varphi$ de l'espace vectoriel $A_{0}$ sur l'espace vectoriel $H_{1}\left(T^{n}, R\right)$.

Soit maintenant $\omega$ une l-forme holomorphe sur $T^{n}$ et soit $L_{\omega}^{\prime}(c)=\int_{c} \omega$ pour tout $c \in H_{1}\left(T^{n}, R\right)$. Soit $L_{\omega}=L_{\omega}^{\prime} \circ \varphi$. $\quad L_{\omega}$ est alors une forme linéaire sur $A_{0}$ à valeurs complexes. Nous allons montrer que $L_{\text {tv }}$ est une forme linéaire sur $A$, c'est-à-dire que l'on a $L_{w}(J x)=\sqrt{ }-1 L_{w}(x)$ pour tout $x \in A_{0}$. Soit $z_{1}, \ldots, z_{n}$ un système de coordonnées complexes de $A$. Soit $Q$ l'espace vectoriel complexe de dimension complexe $n$ de toutes les l-formes holomorphes sur $T^{n}$. Il existe 
une base $\omega_{1}, \ldots, \omega_{n}$ de $\Omega$ telle que $p^{i} \cdot \omega_{k}=d z_{k}(k=1, \ldots, n)$, où $p$ designe la projection canonique de $A$ sur $T^{n}$. Il suffit alors de montrer que $L_{\omega_{k}}(J x)$ $=\sqrt{ }-1 L_{w_{i i}}(x)$ pour tout $x \in A_{0}$ et pour $k=1, \ldots, n$. Soit $\left(a_{1}, \ldots, a_{2 n}\right)$ un système de générateurs de $\Pi$. Alors $\left(a_{1}, \ldots, a_{2 n}\right)$ est une base de $A_{0}$. Alors $L_{\omega_{k}}\left(a_{i}\right)=\int_{\varphi\left(a_{i}\right)} \omega_{k}=\int_{0}^{a_{i}} d z_{k}=z_{k}\left(a_{i}\right)$. Soit $x \in A_{0}$, et soit $x=\sum_{i=1}^{2 n} r_{i} \cdot a_{i}, r_{i} \in R$. On a alors $L_{\omega v_{k}}(x)=\sum_{i=1}^{2 n} r_{i} L_{\omega \omega_{k}}\left(a_{i}\right)=\sum_{i=1}^{2 n} r_{i} \cdot z_{k}\left(a_{i}\right)=z_{k}(x)$. Par suite, $L_{\omega_{k}}(J x)=z_{k}(J x)$ $\sqrt{ }-1 \quad z_{k}(x)=\sqrt{ }-\overline{1} \quad L_{\omega_{k}}(x)$. On a ainsi démontré que $L_{\omega}$ est une forme linéaire sur $A$. Il est clair que $L_{w}=0$ si et seulement si $\omega=0$. Il en suit que l'ap. plication $\omega \rightarrow L_{\omega}$ définit un isomorphisme de $\Omega$ sur le dual $A^{*}$ de $A$.

\section{Fibrés homogènes et connexions holomorphes}

Nous utiliserons dans tout ce qui suit les notations suivantes. $P(M, U, \pi)$ désigne un fibré principal holomorphe de base $M$ compacte, de groupe structural $U$ et de projection $\pi . \quad F_{0}(P)$ (resp. $A_{0}(M)$ ) désigne le plus grand groupe connexe d'automorphismes du fibré holomorphe $P(M, U, \pi)$ (resp. de la variété complexe $M$ ). Si $M=T^{n}$, on a $A_{0}(M)=T^{n}$ (cf. 2. C).

3.1. Un fibré $P(M, U, \pi)$ est dit homogène, si $F_{0}(P)$ est transitif sur les fibres; pour toute couple des points $\left(u_{1}, u_{2}\right)$ de $M$, il existe un $f \in F_{0}(P)$ tel que $f\left(\pi^{-1}\left(u_{1}\right)\right)=\pi^{-1}\left(u_{2}\right)$. Dire que le fibré $P(M, U, \pi)$ est homogène revient à dire que l'image $\pi \cdot F_{0}(P)$ de $F_{0}(P)$ dans $A_{0}(P)$ est transitive sur $M$. La variété $M$ doit être donc homogène complexe. Le but de ce paragraphe est la démonstration du théorème suivant.

THÉORÈME 1. Un fibré principal holomorphe sur un tore complexe est homogène si et seulement s'il possède une connexion holomorphe.

3.2. Démontrons d'abord la proposition suivante (cf. [7]).

Proposition 3.1. Supposons que le fibré $P(M, U, \pi)$ ait une connexion holomorphe et que le groupe $A_{0}(M)$ soit transitif sur $M$. Alors le fibré $P(M, U, \pi)$ est homogène.

Désignons par $a(M)$ l'algèbre de Lie des champs de vecteurs holomorphes sur $M . \quad a(M)$ est l'algèbre de Lie de $A_{0}(M)$. Soit $X \in a(M)$ et soit $X^{*}$ le relèvement de $X$ relatif à la connexion holomorphe $\Gamma$ sur $P$. Alors le champ 
de vecteurs $X^{*}$ est holomorphe (cf. 1 . A) et $R_{u}^{\prime} \cdot X^{*}=X^{*}$ pour tout $a \in U$. Par suite $X^{*}$ appartient à l'algèbre de Lie $\mathfrak{f}(P)$ de $F_{0}(P)$ (cf. 1. A) et on a $\pi^{\prime} \cdot X^{*}$ $=X$. Il en résulte que l'homomorphisme $\pi: F_{0}(P) \rightarrow A_{0}(P)$ est surjectif. Puisque l'on a supposé que $A_{0}(P)$ est transitif sur $M, F_{0}(P)$ est transitif sur les fibres. Le fibré $P(M, U, \pi)$ est donc homogène.

Supposons maintenant que le fibré $P(M, U, \pi)$ soit homogène. Choisissons un point $x_{0} \in P$ et soit $u_{0}=\pi\left(x_{0}\right)$. Soit $\widetilde{B}$ le sous-groupe de $F_{0}(P)$ constitué par les éléments $f \in F_{0}(P)$ tels que $f\left(x_{0}\right) \in \pi^{-1}\left(u_{0}\right)$. Il est chair que $\widetilde{B}$ est fermé dans $F_{0}(P)$. On va montrer que $\widetilde{B}$ est un sous-groupe complexe de $F_{0}(P)$. Soit, en effet, $\mathfrak{b}$ la sous-algèbre de $\mathfrak{f}(P)$ correspondant à $B$. Un champ de vecteurs $X \in \mathfrak{f}(P)$ appartient à $\mathfrak{b}$ si et seulement si $X$ est tangent au fibre $\pi^{-1}\left(u_{0}\right)$. Puisque $\pi^{-1}\left(u_{0}\right)$ est une sous-variété complexe de $P$, si $X$ est tangent à $\pi^{-1}\left(u_{0}\right), I_{P} \cdot X$ l'est aussi. On a ainsi $I_{P} \cdot \mathfrak{b} \subset \mathfrak{b}$ et ceci montre que $\mathfrak{b}$ est une sous-algèbre complexe de $f(P)$ et par suite $\widetilde{B}$ est un sous-groupe complexe de $F_{0}(P)$.

Soit maintenant $b \in \widetilde{B}$. Puisque $b\left(x_{0}\right) \in \pi^{-1}\left(u_{0}\right)$ il existe un et un seul élément $\widetilde{\alpha}(b)$ de $U$ tel que $b\left(x_{0}\right)=x_{0} \cdot \widetilde{\alpha}(b)$. On vérifie facilement que l'application $\tilde{\alpha}$ de $\widetilde{B}$ dans le groupe structural $U$ est un homomorphisme holomorphe de $\widetilde{B}$ dans $U$ (cf. Wang [10]).

D'autre part, $F_{0}(P)$ étant transitif sur les fibres, on peut identifier la base $M$ avec l'espace homogène complexe $F_{0}(P) / \widetilde{B}$ par la correspondance $f(\bmod \widetilde{B})$ $\rightarrow \pi(f)\left(u_{0}\right)$. Considérons le fibré principal holomorphe $F_{0}(P)(M, \widetilde{B}, \widetilde{p}), \widetilde{p}$ désignant la projection canonique de $F_{0}(P)$ sur $M=F_{0}(P) / \widetilde{B}$. Soit $\widetilde{\alpha}$ l'application holomorphe de $F_{0}(P)$ dans $P$ définie par $\widetilde{\alpha}(f)=f\left(x_{0}\right)$. On voit alors que l'application $\tilde{\alpha}: F_{0}(P) \rightarrow P$ et l'homomorphisme $\tilde{\alpha}: \widetilde{B} \rightarrow U$ définissent un homomorphisme du fibré $F_{0}(P)(M, \widetilde{B}, \widetilde{p})$ dans le fibré $P(M, U, \pi)$. Le fibré $P(M, U, \pi)$ est donc associé au fibré $F_{0}(P)(M, \widetilde{B}, \widetilde{p})$ par l'homomorphisme holomorphe $\tilde{\alpha}$ de $\widetilde{B}$ dans $U$.

Supposons maintenant que la base $M$ soit un tore complexe $T^{n}$. On va montrer que $\widetilde{B}$ est un sous-groupe invariant de $F_{0}(P)$. En effet, un élément $f$ de $F_{0}(P)$ appartient à $\widetilde{B}$ si et seulement si $(\pi f)\left(u_{\jmath}\right)=u_{0}$. Puisque $T^{n}=A_{0}\left(T^{n}\right)$ est simplement transitif sur $T^{n}$ et que $\pi f \in A_{0}\left(T^{n}\right)$, cette condition est équivalente à la condition $\pi f=1$. Par suite $\widetilde{B}$ est égal au noyau de l'homomorphisme $\pi: F_{0}(P) \rightarrow A_{0}\left(T^{n}\right)=T^{n}$ et $\widetilde{B}$ est donc un sous-groupe invariant de 
$F_{0}(P)$. Remarquons que l'on a $\pi \cdot F_{0}(P)=T^{n}$. En effet, $\pi \cdot F_{0}(P)$ est contenu dans $T^{n}$ et transitif sur $T^{n}$. Il en résulte immédiatement que $\pi \cdot F_{0}(P)=T^{n}$.

Soit maintenant $G$ le radical de $F_{0}(P) . \quad G$ est, par définition, le plus grand sous-groupe invariant, résoluble et connexe de $F_{0}(P)$. $G$ est un sous-groupe complexe de $F_{0}(P)$. Puisque $T^{n}$ est abélien, l'image dans $T^{n}$ d'un sous-groupe semi-simple connexe de $F_{0}(P)$ se réduit à l'élément neutre. Il résulte de là et du théorème de Levi dans la théorie des algèbres de Lie que l'on a $\pi(G)=T^{n}$. Soit $B=G \cap \widetilde{B}$. Alors $B$ est un sous-groupe invariant, fermé et complexe de $G$ et on a $G / B=F_{0}(P) / \widetilde{B}=T^{n}$. Soit $j$ l'application identique de $G$ dans $F_{0}(P)$. Alors l'application holomorphe $\alpha=\widetilde{\alpha} \circ j$ de $G$ dans $P$ et l'homomorphisme holomorphe $\alpha=\widetilde{\alpha} \circ j$ de $B$ dans $U$ définit un homomorphisme du fibré principal holomorphe $G\left(T^{n}, B, p\right)$ dans le fibré $P\left(T^{n}, U, \pi\right)$. Par suite le fibré homogène $P\left(T^{n}, U, \pi\right)$ est associé au fibre $G\left(T^{n}, B, p\right)$. Nous donnons ici la définition suivante. Soit $G$ un groupe de Lie complexe, résoluble et connexe et soit $B$ un sous-groupe invariant, fermé et complexe de $G$ tel que le groupe quotient $G / B$ soit isomorphe à un tore complexe $T^{n}$. Soit $\alpha$ un homomorphisme holomorphe de $B$ dans un groupe de Lie complexe $U$. Soit $P(T, U, \pi)$ le fibré socié au fibré $G\left(T^{n}, B, p\right.$ ) ( $p$ étant la projection canonique de $G \operatorname{sur} G / B=T^{n}$ ) par l'homomorphisme $\alpha$ de $B$ dans $U$. Le fibré ainsi défini sera appelé de type résoluble.

Il résulte de cette définition et de ce que nous avons déjà dèmontré la proposition suivante.

Proposition 3.2. Tout fibré homogène sur un tore complexe est de type résoluble.

Soit maintenant $G$ un groupe de Lie complexe et connexe et soit $B$ un sous-groupe invariant, fermé et complexe de $G$. Soit $M=G / B$ et soit $p$ la projection canonique de $G$ sur $M$. Nous allons montrer que le fibré principal holomorphe $G(M, B, p)$ possède une connexion holomorphe. Nous identifions l'algèbre de Lie $g$ de $G$ avec l'espace tangent de $G$ à l'élément neutre de $G$. Soit $b$ l'idéal de $g$ correspondant au sous-groupe invariant $B$ de $G$. Puisque $B$ est un sous-groupe complexe, $\mathfrak{b}$ est un sous-espace complexe de $g$. Soit $m$ un sous-espace complexe de $\mathfrak{g}$ tel que $\mathfrak{g}=m+\mathfrak{b}, m \cap \cap \mathfrak{b}=(0)$. Soit $g \in G$ et soit $V_{g}$ le sous-espace de tous les vecteurs verticaux de l'espace tangent $T_{g}$ au point $g$ 
(Un vecteur $X \in T_{g}$ est dit vertical si la projection $p^{\prime} \cdot X$ est nulle). On a alors $V_{g}=L_{g} \cdot \mathfrak{b}$, où $L_{g}$ désigne la translation à gauche par $g$. On va montrer que $V_{g}=R_{g} \cdot \mathfrak{b}, R_{g}$ désignant la translation à droite par $g$. En effet, on a $R_{g} \cdot b$ $=R_{g} \cdot L_{g} \cdot L_{g^{-1}} \mathfrak{b}=L_{g} \cdot$ ad $g^{-1} \cdot \mathfrak{b}$. Puisque $\mathfrak{b}$ est un idéal de $\mathfrak{g}$, on a ad $g^{-1} \cdot \mathfrak{b}=\mathfrak{b}$ et par suite $R_{g} \cdot \mathfrak{b}=L_{g} \cdot \mathfrak{b}=V_{g}$. Posons $Q_{g}=R_{g} \cdot \mathrm{m}$. On a alors $T_{g}=R_{\mathfrak{c}} \cdot \mathfrak{g}=Q_{g}$ $+V_{g}$ (somme directe) et $Q_{g h}=R_{h} Q_{g}$. Le champ de sous-espaces $g \rightarrow Q_{g}$ définit alors une connexion $\Gamma$ sur le fibré $G(M, B, p)$. Il est facile de voir que $\Gamma$ est une connexion holomorphe. Le fibré $G(M, B, p)$ possède ainsi une connexion holomorphe. Comme nous l'avons déjà dit au 2. A, tout fibré associé à un fibré admettant une connexion holomorphe possède lui-même une connexion holomorphe. Il en résulte la proposition suivante.

Proposition 3.3. Tout fibré sur $T^{n}$ de type résoluble possède une connexion holomorphe.

Le théorème 1 résulte immédiatemen des propositions $3.1,3.2$ et 3.3 .

3. 3. Soit $P(M, U, \pi)$ un fibré holomorphe. Soit $\tilde{M}$ le revétement universel de $M$ et soit $I$ le groupe fondamental de $M$. Nous dirons que le fibré $P(M, U, \pi)$ se définit par une représentation du groupe fondamental $\Pi$ de $M$ s'il est associée au fibré principal holomorphe $\tilde{M}(M, \Pi, p)$ par un homomorphisme de $\Pi$ dans $U$. Atiyah [1] a montré que $P(M, U, \pi)$ est défini par une représentation du groupe fondamental de $M$ si et seulement si $P(M, U, \pi)$ possède une connexion holomorphe intégrable (Une connexion est dite intégrable si sa forme de courbure est nulle).

Soit maintenant $P\left(T^{n}, U, \pi\right)$ un fibré homogène sur un tore complexe $T^{n}$. On voit facilement que la condition suivante est nécessaire et suffisante pour que $P\left(T^{n}, U, \pi\right)$ soit définie par une représentation du groupe fondamental de $T^{n}$ : Sous les notations introduites au 3.2, il existe un sous-groupe abélien, connexe et complexe $A$ de $F_{0}(P)$ tel que $A \cap \widetilde{B}$ soit discret et que $F_{0}(P)=A \cdot \widetilde{B}$.

En particulier si $F_{0}(P)$ est lui-même abélien, le fibré homogène $P\left(T^{n}, U, \pi\right)$ est défini par une représentation du groupe fondanental. La proposition suivante est due à Murakami [8].

Proposition 3.4. Soit $P\left(T^{n}, U, \pi\right)$ un fibré homogène sur $T^{n}$. Si le groupe structural $U$ est égal ì $C^{*}$ ou égale à $C$, le groupe $F_{N}(P)$ est abélien et par 
suite dans ces deux cas $P\left(T^{n}, U, \pi\right)$ est défini par une représentation du groupe fondamental de $T^{n}$.

Nous démontrons maintenant deux propositions qui seront utilisées au 5 . La proposition suivante est connue dans le cas où $T^{n}$ est une variété abélienne.

Proposition 3.6. Soit $P\left(T^{n}, C^{*}, \pi\right)$ un fibré possédant une connexion holomorphe. Alors il est défini par une représentation unitaire du groupe fondamental de $T^{n}$.

Soit $T^{n}=A / \Pi$, où $A$ est l'espace vectoriel complexe de dimension complexe $n$ et où $n$ est un sous-groupe discret de rang $2 n$. $I$ est isomorphe au groupe fondamental de $T^{n}$. Soit $\Gamma$ le groupe multiplicatif des représentations du groupe $I r$ dans $C^{*}$. Soit $\Gamma_{0}$ le sous-groupe de $\Gamma$ des représentations unitaires de $I$ dans $C^{*}$. Soit $\gamma$ le sous-groupe de $\Gamma$ de tous les éléments $\mu \in \Gamma$ tels que $\mu(a)=\exp L(a)$ pour tout $a \in \Pi$, où $L$ est une forme linéaire sur $A$. On sait que l'on a $I^{\prime}=I_{0} \times \gamma($ Weil [11], §4). En vue de la proposition 3.4, pour démontrer la proposition 3.5 , il suffit de montrer que le fibré qui est défini par une représentation dans $\gamma$ est trivial. Soit donc $\mu \in \gamma$. Il existe alors une forme linéaire $L$ sur $A$ telle que $\mu(a)=\exp L(a)$ pour tout $a \in \Pi$. Soit $\varphi$ l'isomorphisme canonique de $I$ sur $H_{1}\left(T^{n}, Z\right.$ ) (cf. 2. C). Il existe une l-forme holomorphe $\omega$ sur $T^{n}$ telle que $L(a)=\int_{p(a)} \omega$ pour tout $a \in \Pi$. Soit $p$ la projection canonique de $A$ sur $T^{n}$ et soit $f(x)=\int_{0}^{x} p^{*} \cdot \omega, x \in A$. On a alors $f(x+a)=f(x)+L(a)$ pour tout $x \in A$ et $a \in \Pi$. Soit $\emptyset(x)=\exp f(x)$. Alors $\emptyset$ est une fonction holomorphe sur $A$ telle que $\Phi(x) \neq 0$ pour tout $x \in A$ et que $\emptyset(x+a)=\emptyset(x) \cdot \mu(a)$ pour tout $x \in A$ et $a \in \Pi$. Soit $P\left(T^{n}, C^{*}, \pi\right)$ le fibré défini par la représentation $\mu$ de $I$. L'espace $P$ est le quotient de $A \times C^{*}$ par la relation d'équivalence $(x, \sigma) \sim\left(x+a, \mu(a)^{-1} \cdot \sigma\right)\left(x \in A, \sigma \in C^{*}, a \in I I\right)$. Soit $\rho$ la projection canonique de $A \times C^{*}$ sur $P$. On peut définir une application holomorphe $\Psi$ de $P$ sur $T^{n} \times C^{*}$ par $\Psi(\rho(x, \sigma))=(\not(x), \emptyset(x) \cdot \sigma)\left(x \in A, \sigma \in C^{*}\right)$. L'application $\Psi$ est biunivoque et elle définit un isomorphisme du fibré $P\left(T^{n}\right.$, $\left.C^{*}, \pi\right)$ sur le fibré trivial. La proposition 3.5 est donc démontrée.

Proposition 3.6. Soit $P\left(T^{n}, C^{*}, \pi\right)$ un fibré possédant une connexion holomorphe et soit $E$ le fibré vectoriel de fibre $C$ ássocié à $P\left(T^{n}, C^{*}, \pi\right)$. Si le fibré vectoriel $E$ possède une section holomorphe non nulie, E est trivial. 
D'après la proposition $3.5^{\circ}, E$ est le fibré de fibre $C$ associé au fibré $A\left(T^{n}, \Pi, p\right)$ par une représentation unitaire $\alpha$ de $\Pi$ dans $C^{*}$. $E$ est l'espace. quotient de $A \times C$ par la relation d'équivalence $(x, \xi) \sim\left(x+a, \alpha(a)^{-1} \cdot \xi\right)$ $(x \in A, \xi \in C, a \in I I)$. Une section holomorphe $s \neq 0$ de $E$ détermine une fonction holomorphe $f$ sur $A, f \neq 0$, telle que $f(x)=\alpha(a) \cdot f(x+a)$ pour tout $x \in A$ et $a \in \Pi$. On a alors $|f(x)|=|f(x+a)|$. Il en résulte que $f$ est bornée et par suite $f$ est constante: $f(x) \equiv f_{0}, f_{0} \neq 0$. On a alors $f_{0}=\alpha(a) \cdot f_{0}$ pour tout $a \in \Pi$ et par suite $\alpha=1$. Il en résulte immédiatement que le fibré $E$ est trivial.

\section{Décomposition en facteurs indécomposables}

4.1. Soit $P(M, G L(m, C), \pi)$ le fibré principal holomorphe de groupe structural $G L(m, C)$ et soit $E$ le fibré vectoriel holomorphe de fibre $C^{\prime n}$ associé à $P(M, G L(m, C), \pi)$. Nous dirons que le fibré vectoriel $E$ possède une connexion holomorphe (resp. $E$ est homogène) si $P(M, G L(m, C), \pi$ ) en possède une (resp. $P(M, G L(m, C), \pi)$ est homogène).

Dans ce paragraphe nous démontrons le théorème suivant.

Theoreme 2. Soit E un fibré vectoriel holomorphe sur une variété compacte et connexe $M$ et soit $E=E_{1} \oplus \ldots \oplus E_{k}$ une décomposition de $E$ en facteurs indécomposables. Alors E est homogène si et seulement si chaque fasteur l'est.

Il résulte des théorèmes 1 et 2 le corollaire suivant.

Corollaire. Soit $E$ un fibré vectoriel holomorphe sur un tore complexe et soit $E=E_{1} \oplus \ldots \oplus E_{k}$ une décomposition de $E$ en facteurs indécomposables. Alors $E$ possede une connexion holomorphe si et seulement si chaque facteur en possède une.

4.2. Rappelons d'abord la proposition suivante due à Atiyah [3].

Proposition 4.1. Soient $E=E_{1} \oplus \ldots \oplus E_{k}$ et $E=E_{1}^{\prime} \oplus \ldots \oplus E_{l}^{\prime}$ deux décompositions de $E$ en facteurs indécomposables. On a alors $k=l$ et il existe une permutation o de $(1, \ldots, k)$ telle que $E_{i} \cong E_{\Im_{(i)}}^{\prime}$ pour $i=1,2, \ldots, k$.

Remarque. Sous les notations dans la proposition 4.1, il est clair qu'il existe un automorphisme $g$ de $E$ (cf. 2. B) tel que 1 ) $g \cdot E_{i}=E_{3(i)}^{\prime}$ pour $i=1$, $\ldots, k, 2)$ la projection de $g$ sur la base est l'automorphisme identique de la base. 
Lemme 4.1. Soit $E$ un fibré vectoriel sur $M$ de fibre $C^{m}$ et soit $E_{1}$ un sous-fibré vectoriel de E. Soit $f$ un automorphisme de $E$. Alors $f\left(E_{1}\right)$ est aussi un sous-fibré vectoriel de E.

D'après la définition d'un sous-fibré vectoriel de $E$, il existe un atlas $\left\{\varphi_{i}, U_{i}\right\}_{i \subseteq l}$ de $E$ et un sous-espace complexe $F_{1}$ de $C^{m}$ tels que 1$)$ les fonctions de changement $s_{i j}(i, j \in I)$ satisfont à la condition $s_{i j}(u) \cdot F_{1} \subset F_{1}$ pour tout $\left.u \in U_{i} \cap U_{j}, 2\right) E=\bigcup_{i \in I} \varphi_{i}\left(U_{\imath} \times F_{1}\right)$.

Soit $\vec{f}$ la projection de $f$ (cf. 2. B). Définissons les applications $\varphi_{i}^{\prime}: \bar{f}\left(U_{i}\right)$ $\times C^{m} \rightarrow \pi^{-1}\left(\bar{f}\left(U_{i}\right)\right)$ ( $\pi$ étant la projection de $E$ sur la base) comme suit: $\varphi_{i}^{\prime}(\bar{f}(u), \xi)=f\left(\varphi_{i}(u, \xi)\right)\left(u \in U_{i}, \xi \in C^{m}\right)$. Il est facile de voir que $\left\{\varphi_{i}^{\prime}, \bar{f}\left(U_{i}\right)\right\}_{i \in I}$ est un atlas de $E$. Les fonctions de changement de cet atlas, $s_{\imath \jmath}^{\prime}: \bar{f}\left(U_{i}\right) \cap \bar{f}\left(U_{j}\right)$ $\rightarrow G L(m, C)$, sont telles que $s_{i j}^{\prime}(\bar{f}(u))=s_{i j}(u)$ pour tout $u \in U_{i} \cap U_{j}$. Par suite on a $s_{i j}^{\prime}(v) \cdot F_{1} \subset F_{1}$ pour tout $v \in \bar{f}\left(U_{i}\right) \cap \bar{f}\left(U_{j}\right)$. De plus, on a $f\left(E_{1}\right)=\bigcup_{i \in l}^{C_{i}^{\prime}}\left(\bar{f}\left(U_{i}\right)\right.$ 火 $\left.F_{1}\right) . \quad f\left(E_{1}\right)$ est donc un sous-fibré de $E$.

Le iemme suivant est facile à démontrer.

Lemme 4.2. Soient $E_{1}$ et $E_{2}$ deux sous-fibrés vectoriels de $E$ et soit $f$ un automorphisme de E. Alors

a) si $E_{1} \cong E_{2}$, on a $f\left(E_{1}\right) \cong f\left(E_{2}\right)$,

b) si $E=E_{1} \oplus E_{2}$, on a $E=f\left(E_{1}\right) \oplus f\left(E_{2}\right)$,

c) si $E_{1}$ est indécomposable, $f\left(E_{1}\right)$ l'est aussi.

Lemme 4.3. Soit $\theta$ un homomorphisme d'un groupe de Lie connexe $C$ dans un groupe fini $H$. Alors $\theta$ est l'application constante de $G$ sur l'élément neutre de $H$.

Soit, en effet, $n$ l'ordre de $H$ et soit $N$ le noyau de l'homomorphisme $\theta$. On a alors $g^{n} \in N$ pour tout $g \in G$. Soit $x(t)(-\infty<t<+\infty)$ un sous-groupe à un paramétre de $G$. On a $x(t)=x(t / n)^{n}$ pour tout $t$ et par suite $N$ contient tout sous-groupe à un paramètre de $G$. Puisque $G$ est connexe, ceci implique $N=G$.

Démonstration du théorème 2. Soit $E$ un fibré vectoriel holomorphe sur $M$ compacte et connexe, de fibre $C^{m}$ et soit $P(M, G L(m, C), \pi)$ le fibré principal associé à $E$. Nous identifions, comme au 2. B, le groupe d'automorphismes de $P(M, G L(m, C), \pi)$ avec celui de $E$. Soit $E=E_{1}^{1} \oplus \ldots \oplus E_{n_{1}}^{1} \dot{\oplus} E_{1}^{2} \oplus$ 
$\ldots \oplus E_{n_{2}}^{2} \oplus \ldots \oplus E_{1}^{r} \oplus \ldots \oplus E_{n_{r}}^{r}$ une décomposition de $E$ en facteurs indécomposables. Supposons que $E_{j}^{i} \cong E_{l}^{i}\left(i=1, \ldots, r ; j, l=1, \ldots, n_{i}\right)$ et que $E_{j}^{i} \neq E_{l}^{k}(i \neq k)$. Soit $P_{j}^{i}$ le fibré principal associé à $E_{j}^{i}$. Soit maintenant $F_{0}(P)$ la composante connexe de l'élément neutre du groupe $F(P)=F(E)$ et soit $f \in F_{0}(P)$. On a alors $E=f\left(E_{1}^{1}\right) \oplus \ldots \oplus f\left(E_{n_{r}}^{r}\right)$ et chaque sous-fibré $f\left(E_{j}^{i}\right)$ est indécomposable. De plus, $f\left(E_{j}^{i}\right) \cong f\left(E_{j}^{i}\right)$ et $f\left(E_{j}^{i}\right) \neq f\left(E_{l}^{k}\right)(i \neq k)$. D'après la proposition 4.1 , il existe une permutation of de $(1, \ldots, r)$ telle que $f\left(E_{j}^{i}\right)$ $\cong E_{j^{\prime}}^{f^{(i)}}\left(i=1, \ldots, r ; j=1, \ldots, n_{i}\right)$. Il est facile de voir que $f \rightarrow \sigma_{f}$ est un homomorphisme de $F_{0}(P)$ dans le groupe de permutations de $(1, \ldots r)$. Il résulte du lemme 4.3 que $\sigma_{f}=1$ pour tout $f \in F_{0}(P)$. On a donc montré que $f\left(E_{j}^{i}\right) \cong E_{j}^{i}$ pour tout $f \in F_{0}(P)$. D'après la remarque à la proposition 4.1 , il existe un automorphisme $g$ de $E$ tel que $g \cdot f\left(E_{j}^{i}\right)=E_{j}^{i}(i=1,2, \ldots, r ; j=1$, $\ldots, n_{i}$ ) et que la projection de $g$ sur la base $M$ soit l'automorphisme identique de $M$. Soit $f_{i j}$ la restriction de $g \cdot f$ au sous-fibré $E_{j}^{i}$. Alors $f_{i j}$ est un automorphisme du fibré $E_{j}^{i}$ et la projection de $f_{i j}$ sur $M$ est égal à celle de $f$. Soit $F\left(P_{j}^{i}\right)$ le groupe d'automorphismes du fibré $P_{j}^{i}$. Il résulte de ce que nous avons montré et du $2 . \mathrm{B}$ que $\pi \cdot F\left(P_{j}^{i}\right) \supset \pi \cdot F(P)$. Supposons maintenant que le fibré vectoriel $E$ est homogène. Alors $\pi \cdot F_{0}(P)$ est transitif sur $M$ et par suite $\pi \cdot F\left(P_{j}^{i}\right)$ est aussi transitif sur $M$. Puisque l'on a supposé que $M$ set connexe, $\pi \cdot F_{0}\left(P_{j}^{i}\right)$ est transitif sur $M{ }^{4}{ }^{\prime}$ Les fibrés vectoriels $E_{j}^{i}$ sont donc homogènes. La réciproque est triviale et le théorème 2 est donc démontré.

\section{Réduction du groupe structural}

Dans ce paragraphe et dans le paragraphe qui suit nous utiliserons les notations suivantes. $T^{n}$ désigne un tore complexe de dimension complexe $n$.

$H\left(T^{n}, m\right)$ : L'ensemble des fibrés principaux holomorphes sur $T^{n}$ de groupe structural $G L(m, C)$.

$\boldsymbol{H}\left(\boldsymbol{T}^{n}, \boldsymbol{m}\right)$ : L'ensemble des classes d'équivalence de fibrés dans $H\left(T^{n}, m\right)$.

$E\left(T^{n}, m\right)$ : L'ensemble des fibrés principaux holomorphes sur $T^{n}$ de groupe structural $G L(m, C)$ qui sont indécomposables et qui possèdent une connexion holomorphe.

4) On a utilisé ici le fait suivant: Si un groupe de Lie $G$ opère transitivement sur une variété connexe $M$ et si $G$ et $M$ satisfont au second axiome de dénombrabilité, alors la composante connexe de l'élément neutre de $G$ est aussi transitive sur $M$. 
$\boldsymbol{E}\left(\boldsymbol{T}^{n}, \boldsymbol{m}\right)$ : Le sous ensemble de $\boldsymbol{H}\left(\boldsymbol{T}^{n}, \boldsymbol{m}\right)$ des classes qui contiennent des fibrés dans $E\left(T^{n}, m\right)$.

$N(m, C)$ : Le sous-groupe de $G L(m, C)$ des matrices de la forme

$$
\left(\begin{array}{lllll}
1 & & & & \\
& 1 & & & * \\
& 0 & \cdot & & \\
& & & \cdot & \\
& & & & 1
\end{array}\right) .
$$

Soit $N^{\prime}\left(T^{n}, m\right)$ l'ensemble des fibrés principaux holomorphes sur $T^{n}$ de groupe structural $N(m, C)$ admettant une connexion holomorphe. L'application identique $j$ de $N(m, C)$ dans $G L(m, C)$ induit une application $j$ de $N^{\prime}\left(T^{n}, m\right)$ dans $H\left(T^{n}, m\right)$. Tout fibré dans $j \cdot N^{\prime}\left(T^{n}, m\right)$ possède une connexion holomorphe.

$N\left(T^{n}, m\right)$ : L'ensemble des fibrés dans $j \cdot N^{\prime}\left(T^{n}, m\right)$ qui sont indécomposables; $N\left(T^{n}, m\right)=E\left(T^{n}, m\right) \cap j \cdot N^{\prime}\left(T^{n}, m\right)$.

$\boldsymbol{N}\left(\boldsymbol{T}^{n}, \boldsymbol{m}\right):$ Le sous-ensemble de $\boldsymbol{H}\left(\boldsymbol{T}^{n}, \boldsymbol{m}\right)$ des classes qui contiennent des fibrés dans $N\left(T^{n}, m\right)$.

On a $H\left(T 0^{n}, m\right) \supset E\left(T^{n}, m\right) \supset N\left(T^{n}, m\right)$.

5.1. Le but de ce paragraphe est la démonstration du théorème suivant.

ThéoRème 3. L'application de $E\left(\boldsymbol{T}^{n}, \mathbf{1}\right) \times \boldsymbol{N}\left(\boldsymbol{T}^{n}, \boldsymbol{m}\right)$ dans $\boldsymbol{H}\left(\boldsymbol{T}^{n}, \mathbf{m}\right)$ définie par $(\xi, \eta) \rightarrow \xi \otimes \eta$ donne une application biunivoque de $E\left(T^{n}, 1\right) \times N\left(T^{n}, m\right)$ sur $E\left(\boldsymbol{T}^{n}, \mathbf{m}\right)$.

Corollaire. Tout fibré principal $P\left(T^{n}, G L(m, C), \pi\right)$ qui possède une connexion holomorphe est topologiquement trivial.

En effet, en vue du corollaire du théorème 2, on peut supposer que $P\left(T^{n}, G L(m, C), \pi\right)$ est indécomposable. D'apres le théorème 3 il existe un $P_{1} \in E\left(T^{\prime}, 1\right)$ et un $P_{2} \in N\left(T^{n}, m\right)$ tels que $P \cong P_{1} \otimes P_{2}$. Il est clair que tout fibré dans $N\left(T^{n}, m\right)$ est topologiquement trivial, car le groupe $N(m, C)$ est homéomorphe à l'espace numérique de certaine dimension. D'autre part, le fibré $P_{1}$ de groupe structural $C^{*}$ possède une connexion holomorphè et par suite sa classe de Chern est nulle (cf. Atiyah [1]). Alors $P_{1}$ est topologiquement trivial (cf. Hirzebruch [4], p.64). Les fibrés $P_{1}$ et $P_{2}$ sont donc topologiquement triviaux et par suite $P$ l'est aussi. 
5.2. Etablissons d'abord quelques lemmes qui sont nécessaires pour la démonstration du théorème 3 .

LeMme 5.1. Soit $B$ un groupe de Lie complexe et résoluble tel que le groupe quotient $B / B_{0}$ soit un groupe abélien, où $B_{0}$ désigne la composante connexe de l'élément neutre de $B$. Soit a une représentation linéaire holomorphe de $B$ dans un espace vectoriel complexe F telle que, pour tout $g \in B, \alpha(g)$ n'ait qu'une valeur propre. Alors on peut trouver une suite de sous-espaces complexes de $F$

$$
F_{1} \subset F_{2} \subset \ldots \subset F_{m-1} \subset F_{m}=F
$$

telle que la dimension complexe de $F_{k}$ soit égale $\grave{a} k(k=1,2, \ldots, m)$ et telle que $\alpha(g) \cdot F_{k} \subset F_{k}$ pour tout $g \in B$ et $k=1,2, \ldots, m$.

Nous démontrons le lemme par récurrence sur la dimension (complexe) de $F$. Si la dimension de $F$ est égale à 1 , le lemme est trivial. Supposons donc que le lemme soit démontré pour toute représentation linéaire holomorphe de $B$, vérifiant la condition énoncée dans le lemme, dans un espace vectoriel complexe de dimension plus petite que $m$. Soit $F$ un espace vectoriel complexe de dimension $m$ et soit $\alpha$ une représentation linéaire holomorphe de $B$ dans $F$ telle que, pour tout $g \in B, \alpha(g)$ n'ait qu'une valeur propre. Soit b l'algèbre de Lie de $B$ et soit $\alpha^{\prime}$ la représentation linéaire de $b$ induite par la représentation $\alpha$ de $B$. Une forme linéaire $\rho$ sur $b$ sera dite le poids de la représentation $\alpha^{\prime}$ s'il existe un élément $\xi \neq 0$ dans $F$ tel que $\alpha^{\prime}(X) \cdot \xi=\rho(X) \cdot \xi$ pour tout $X \in \mathfrak{b}$. Il résulte d'un théorème de Lie et de l'hypothése sur la représentation $\alpha$ qu'il existe un et un seul poids $\rho$ de la représentation $\alpha^{\prime}$. Soit $F^{(1)}$ le sousespace de $F$ de tous les éléments $\xi \Subset F$ tels que $\alpha^{\prime}(X) \cdot \xi=\rho(X) \cdot \xi$ pour tout $X \in \mathfrak{b}$. Alors $F^{(1)} \neq(0)$. Soit $\xi \in F^{(1)}$ et soit $\xi \neq 0$. Soit $g \in B$. Alors $\alpha^{\prime}(X)$ $(\alpha(g) \cdot \xi)=\alpha(g) \cdot \alpha\left(g^{-1}\right) \cdot \alpha^{\prime}(X) \cdot \alpha(g) \cdot \xi=\rho\left(\operatorname{ad~} g^{-1} \cdot X\right)(\alpha(g) \cdot \xi)$. Ceci montre que la forme linéaire $\rho^{\prime}$ sur $\mathfrak{b}$ définie par $\rho^{\prime}(X)=\mu\left(\operatorname{ad} g^{-1} \cdot X\right)$ est un poids de la représentation $\alpha^{\prime}$. Puisque $\alpha^{\prime}$ n'a qu'un poids $\rho$, on a $\rho^{\prime}=\rho$ et par suite $\alpha^{\prime}(X) \cdot(\alpha(g) \cdot \xi)=\rho(X)(\alpha(g) \cdot \xi)$. Ceci montre que $F^{(1)}$ est stable par $B$. Supposons d'abord que la dimension de $F^{(1)}$ soit plus petite que $m$. Les représentations de $B$ dans les espaces vectoriels $F^{(1)}$ et $F / F^{(1)}$ induites par $\alpha$ satisfont à la condition enoncée dans le lemme. L'hypothèse de récurrence établie alors le lemme. Supposons donc que $F^{1:}=F$. On a alors $\alpha^{\prime}(X) \cdot \xi$ $=\rho(X) \cdot \xi$ pour tout $X \in \mathfrak{b}$ et $\xi \in F$. Tout $\alpha(g), g \in B_{0}$, s'écrit alors $\alpha(g)$ 
$=\lambda(g) \cdot I$, où $\lambda(g) \in C^{*}$ et $I$ désigne l'automorphisme identique de $F$. Soit $f$ et $g$ des éléments de $B$. Puisque l'on a supposé que $B / B_{0}$ est abélien, on a $f \cdot g \cdot f^{-1} \cdot g^{-1} \in B_{0}$. Posons $\lambda\left(f \cdot g \cdot f^{-1} \cdot g^{-1}\right)=\lambda$. On a alors $\alpha(f) \cdot \alpha(g)$ $=\lambda \cdot \alpha(g) \cdot \alpha(f)$. Par l'hypothèse $\alpha(f)$ n'a qu'une valeur propre et soit $\mu$ la valeur propre de $\alpha(f)$. Il existe alors un $\xi \in F, \xi \neq 0$, tel que $\alpha(f) \cdot \xi=\mu \cdot \xi$. Alors $\alpha(f)(\alpha(g) \cdot \xi)=\lambda \cdot \alpha(g)(\alpha(f) \xi)=\lambda \cdot \mu \cdot(\alpha(g) \cdot \xi)$. Ceci montre que $\lambda \cdot \mu$ est aussi une valeur propre de $\alpha(f)$. On a donc $\mu=\lambda \cdot \mu$ et par suite $\lambda=1$, car $\mu \neq 0$. Il résulte de là et de ce que nous avons déjà montré que l'on a $\alpha(f) \cdot \alpha(g)=\alpha(g) \cdot a(f)$ pour tout $f, g \in B$. Donc le groupe $\alpha(B)$ est abélien. Il est alors clair qu'il existe une suite de sous-espaces $F_{1} \subset F_{2} \subset \ldots \subset F_{m}=F$ vérifiant les conditions réquises. Le lemme est ainsi établi.

Lемме 5.2. Soit $E$ un fibre vectoriel holomorphe sur $T^{n}$ de fibre $C$ possédant une connexion holomorphe et soit $F$ un fibré vectoriel holomorphe sur $T^{n}$ de fibre $C^{m}$ tel que le groupe structural de $F$ se réduise au sous-groupe $N(m, C)$ de $G L(m, C)$. Si le fibré vectoriel $E \otimes F$ a une section holomorphe non nulle, on a $E=I, I$ désignant le fibré vectoriel trivial de fibre $C$.

Nous démontrons le lemme par récurrence sur la dimension $m$ de la fibre de $F$. Si $m=1$, on a $F=I$, car le groupe $N(m, C)$ se réduit à l'élément neutre de $G L(m, C)$. Le lemme résulte alors de la proposition 3.6. Supposons donc que le lemme soit démontré dans le cas où la dimension de la fibre de $F$ est plus petite que $m$. Soit $F$ un fibré vectoriel vérifiant les conditions énoncées dans le lemme. Puisque le groupe structural de $F$ se réduit à $N(m, C)$ il existe une suite exacte des fibrés vectoriels $0 \rightarrow F_{1} \rightarrow F \rightarrow I \rightarrow 0$. Ici le groupe structural de $F_{1}$ se réduit à $N(m-1, C)$. On a alors la suite exacte $0 \rightarrow E \otimes F_{1}$ $\vec{\alpha} E \otimes F \vec{\beta} E \rightarrow 0$.

Par l'hypothése du lemme, le fibré vectoriel $E \otimes F$ a une section holomorphe $s \neq 0$. Alors $\beta \circ s$ est une section holomorphe de $E$. Si $\beta \circ s \neq 0$, on a $E=I$ d'aprés la proposition 3.6. Supposons donc $\beta^{\circ} s=0$. On a alors $s(x) \in \alpha\left(E \otimes F_{1}\right)$ pour tout $x \in T^{n}$. Alors $s$ définit une section holomorphe non nulle de $E \otimes F_{1}$. Par l'hypothèse de récurrence on a $E=I$. Le lemme est donc établi.

Lemme 5.3. Soient $E_{1}$ et $E_{2}$ des fibrés vectoriels holomorphes sur $T^{n}$ de fibre $C$ possédant une connexion holomorphe. Soient $F_{1}$ es $F_{2}$ des fibrés vectoriels holomorphes sur $T^{n}$ de fibre $C^{m}$ tels que le groupe structural se réduise au sous- 
groupe $N(m, C)$ de $G L(m, C)$. Si $E_{1} \otimes F_{1} \cong E_{2} \otimes F_{2}$ on a $E_{1} \cong E_{2}$ et $F_{1} \cong F_{2}$.

Il résulte de $E_{1} \otimes F_{1} \cong E_{2} \otimes F_{2}$ que $F_{1} \cong E \otimes F_{2}$, où $E=E_{1}^{*} \otimes E_{2}, E_{1}^{*}$ désigant le dual de $E_{1}$. Puisque le groupe structural de $F_{1}$ se réduit à $N(m, C)$, le fibré $F_{1}$ possède une section holomorphe non nulle. Il résulte de $F_{1} \cong E \otimes F_{2}$ et du lemme 5.4 que $E=I$. Alors $E_{1} \cong E_{2}$ et $F_{1} \cong F_{2}$.

Démonstraìion du théorème 3. Soient $\xi \in \boldsymbol{E}\left(\boldsymbol{T}^{n}, \mathbf{1}\right)$ et $\eta \in \boldsymbol{N}\left(\boldsymbol{T}^{n}, \boldsymbol{m}\right)$. Montrons d'abord que $\xi \otimes \eta \boldsymbol{\xi} \in \boldsymbol{E}\left(\boldsymbol{T}^{n}, \boldsymbol{m}\right)$. Soit $E$ (resp. $F$ ) le fibré vectoriel associé à un fibré principal dans la classe $\xi$ (resp. dans la classe $\eta$ ). D'après la définition de $N\left(T^{n}, m\right), F$ est indécomposable et par suite $E \otimes F$ l'est aussi. Les fibrés $E$ et $F$ possèdent une connexion holomorphe et par suite ils sont homogènes. Il est facile de voir que le fibré $E \otimes F$ est aussi homogène. Le fibré $E \otimes F$ possède donc une connexion holomorphe. Il résulte de ce que nous avons montré que $\hat{\xi} \otimes) \eta E\left(\boldsymbol{T}^{n}, \boldsymbol{m}\right)$. Nous allons montrer maintenant que l'application de $\boldsymbol{E}\left(\boldsymbol{T}^{n}, \mathbf{1}\right) \times \boldsymbol{N}\left(\boldsymbol{T}^{n}, \boldsymbol{m}\right)$ dans $\boldsymbol{E}\left(\boldsymbol{T}^{n}, \boldsymbol{m}\right)$ donnée par $(\xi, \eta) \rightarrow \xi \otimes \eta$ est surjectif. Soit donc $\zeta \in E\left(\boldsymbol{T}^{n}, \boldsymbol{m}\right)$ et soit $P\left(T^{n}, G L(m, C), \pi\right)$ un fibré principal dans la classe $\zeta$. Alors le fibré $P$ possède une connexion holomorphe et est indécomposable. Soit $G$ le radical du groupe $F_{0}(P)$ et soit $x_{0} \in P$ (pour ce qui suit, cf. 3.2). Soit $B$ le sous-groupe de $G$ des éléments $b \in G$ tels que $b\left(x_{0}\right) \in \pi^{-1}\left(u_{0}\right)$, où $u_{0}=\pi\left(x_{0}\right)$. On sait que l'homomorphisme $\pi: F_{0}(P) \rightarrow A_{0}\left(T^{n}\right)$ $=T^{n}$ induit l'homomorphisme (que l'on désigne encore par $\pi$ ) de $G \operatorname{sur} T^{n}$ et que $B$ coïncide avec le noyau de ce homomorphisme. On a par suite $G / B=T^{n}$. Le fibré $P$ est associé au fibré principal holomorphe $G\left(T^{n}, B, p\right.$ ) ( $p$ étant la projection canonique de $G$ sur $T^{n}=G / B$ ) par un homomorphisme holomorphe $\alpha$ de $B$ dans $G L(m, C)$. L'homomorphisme $\alpha$ est défini comme suit: pour tout $b \in B, \alpha(b)$ est un élément de $G L(m, C)$ tel que $b\left(x_{0}\right)=x_{0} \cdot \alpha(b)$. Soit maintenant $E$ le fibré vectoriel de fibre $C^{m}$ associé à $P$. Identifions, comme au 2. B, le groupe $F(P)$ avec le groupe d'automorphismes $F(E)$ de $E$. Le sousgroupe $G$ de $F(P)$ peut être considéré comme un sous-groupe de $F(E)$. $B$ est alors le sous-groupe de $G$ des éléments $b \in G$ tels que ses projections sur la base $T^{n}$ soient l'automorphisme identique de la variété $T^{n}$. Nous allons démontrer que $\alpha(b)$ n'a qu'une valeur propre pour tout $b \in B$. L'espace $E$ est le quotient de $P \times C^{m}$ par la relation d'équivalence $(x, \xi) \sim\left(x \cdot a, a^{-1} \cdot \xi\right)\left(x \in P, \xi \in C^{m}, a\right.$ $\in G L(m, C))$. Soit $\rho$ la projection canonique de $P \times C^{m}$ sur $E$. Soit $E_{u_{0}}$ la 
fibre de $E$ au-dessus de $u_{0}=\pi\left(x_{0}\right)$. Désignons par $\bar{x}_{0}$ l'isomorphisme de $C^{\prime \prime}$ sur $E_{u_{0}}$ donné par $\bar{x}_{0} \bullet \xi=\rho\left(x_{0}, \hat{\xi}\right)$. Soit maintenant $b \in B$. On a alors $b \cdot E_{u_{0}} \subset E_{u_{0}}$. Puisque $b$ est un automorphisme de $E, b$ induit un automorphisme $\beta(b)$ de l'espace vectoriel $E_{u_{0}}$. On a donc $b\left(\bar{x}_{0} \bullet \xi\right)=\beta(b)\left(\bar{x}_{0} \bullet \xi\right)$ pour tout $\xi \in C^{m}$. D'autre part, $b\left(\bar{x}_{0} \cdot \xi\right)=b \cdot \rho\left(x_{0}, \xi\right)=\rho\left(b\left(x_{0}\right), \xi\right)=\rho\left(x_{0} \cdot \alpha(b), \xi\right)=\rho\left(x_{0}, \alpha(b) \cdot \xi\right)$ $=\bar{x}_{0} \cdot(\alpha(b) \cdot \xi)\left(\right.$ cf. 2. B). Par suite on a $\alpha(b)=\bar{x}_{0}^{-1} \cdot \beta(b) \cdot \bar{x}_{0}$ pour tout $b \in B$. Or, le fibré vectoriel $E$ étant indécomposable, l'automorphisme $\beta(b)$ de l'espace vectoriel $E_{u_{0}}$ n'a qu'une valeur propre (Atiyah [1], p. 201. Prop. 16). Par suite $\alpha(b)$ n'a qu'une valeur propre. Soit maintenant $B_{0}$ la composante connexe de l'élément neutre de $B$. Alors le groupe $G / B_{0}$ est un revêtement de $G / B=T^{n}$ et donc $G / B_{0}$ est abélien. Par suite $B / B_{0}$ est abélien. D'après le lemme 5.1 il existe un élément $c \in G L(m, C)$ tel que

$$
c \cdot \alpha(b) \cdot c^{-1}=\left(\begin{array}{ccc}
/ \ell(b) & & \\
\cdot & & * \\
0 & \cdot & \\
& & \mu(b)
\end{array}\right)
$$

pour tout $b \in B$. Soit $\alpha^{\prime}$ la représentation de $B$ telle que $\alpha^{\prime}(b)=c \cdot \alpha(b) \cdot c^{-1}$ pour tout $b \in B$ et soit $P^{\prime}\left(T^{n}, G L(m, C), \pi\right)$ le fibré associé à $G\left(T^{\prime \prime}, B, p\right)$ par la représentation $\alpha^{\prime}$. On a alors $P \cong P^{\prime}$. On peut donc supposer, sans gêner la généralité, que $c=I$ et donc que

$$
\alpha(b)=\left(\begin{array}{ccc}
\mu(b) & & \\
\cdot & & * \\
0 & \cdot \\
\mu(b)
\end{array}\right) .
$$

$b \rightarrow \not l(b)$ est un homomorphisme holomorphe $"$ de $B$ dans $C^{*}$. Soit $\varepsilon$ l'homomorphisme holomorphe de $B$ dans $G L(\mathrm{~m}, C)$ défini par $\varepsilon(b)=\| l(b)^{-1} \cdot \alpha(b)$. On a alors $\varepsilon(b) \in N(m, C)$ pour tout $b \in B$ et $\alpha=\mu x, \varepsilon$. Puisque $\varepsilon(b) \in N(m, C)$ pour tout $b \in B$, on peut considérer $\varepsilon$ comme un homomorphisme holomorphe $\varepsilon^{\prime}$ de $B$ dans $\left.N, m, C\right)$. Soit $j$ l'application identique de $N(m, C)$ dans $G L(m, C)$. On a alors $\varepsilon=j \circ \varepsilon^{\prime}$. Soit $P_{2}$ (resp. $P_{2}^{\prime}$ ) le fibré principal de groupe structural $G L(m, C)$ (resp. $N(m, C)$ ) associé à $G\left(T^{n}, B, p\right)$ par l'homomorphismes $\varepsilon$ (resp. $\left.\varepsilon^{\prime}\right)$. Alors $P_{2}$ est associé à $P_{2}^{\prime}$ par l'application identique $j$ de $N(m, C)$ dans $G L(m, C)$. D'autre part, d'après la proposition 3.3 , le fibré $P_{2}^{\prime}$ possède une. 
connexion holomorphe. Soit maintenant $P_{1}$ le fibré principal de groupe structural $C^{*}$ associé à $G\left(T^{2}, B, p\right)$ par l'homomorphisme /. D'aprés la proposition 3.3, $P_{1}$ possède une connexion holomorphe. Puisque $\alpha=\mu\left(\hat{x} \varepsilon\right.$, on a $P \cong P_{1} \otimes P_{2}$. Le fibré $P$ étant indécomposable, le fibré $P_{2}$ l'est aussi. Il résulte de ce que nous avons montré que $P_{1} \in E\left(T^{\eta}, 1\right)$ et $P_{2} \in N\left(T^{n}, m\right)$. Soient $\xi$ et $\eta$ les classes contenant les fibrés $P_{1}$ et $P_{2}$ respectivement. Alors $\xi \in E\left(\boldsymbol{T}^{n}, 1\right)$, $\eta \in N\left(\boldsymbol{T}^{n}, \boldsymbol{m}\right)$ et $;=\xi(\theta)$. Nous avons donc démontré que l'application de $\boldsymbol{E}\left(\boldsymbol{T}^{n}, \mathbf{1}\right) \times \boldsymbol{N}\left(\boldsymbol{T}^{n}, \boldsymbol{m}\right)$ dans $\boldsymbol{E}\left(\boldsymbol{T}^{n}, \boldsymbol{m}\right)$ donnée par $(\xi, \eta) \rightarrow \xi \otimes \eta$ est surjective. Le fait que cette application est biunivoque résulte immédiatement du lemme 5.3. Le théorème 3 est ainsi démontré.

\section{Le cas $m=2$}

Nous étudions ici le cas où le groupe structural est $G L(2, \mathrm{C})$. Nous utiliserons les notations introduites au $\bar{j}$.

6. 1. Démontrons d'abord le théorème suivant.

THÉORÈME 4. Tout fibré principal holomorphe sur un tore complexe, de groupe structural $G L(2, \mathrm{C})$ qui possède une connexion holomorphe se définit par une représentation du groupe fondamental de $T^{n}$ dans $G L(2, \mathrm{C})$.

En effet, le groupe $N(2, \mathrm{C})$ est isomorphe au groupe $C$. Alors le théorème 4 est une conséquence immédiate des théorème 2 et 3 et de la proposition 3.4.

THÉORÈME $5 . \quad 1 l$ existe une correspondance biunivoque bien déterminée entre $\boldsymbol{E}\left(\boldsymbol{T}^{n}, 2\right)$ et la variété $T^{\prime \prime} \times P^{n-1}$, où $T^{\prime \prime}$ désigne la variété de Picard de $T^{n}$ et oì $P^{n-1}$ désigne l'espace projectif complexe de dimension complexe $n-1$

D'après le théorème 3 on a $\boldsymbol{E}\left(\boldsymbol{T}^{n}, \mathbf{2}\right)=\boldsymbol{E}\left(\boldsymbol{T}^{n}, \mathbf{1}\right) \times \boldsymbol{N}\left(\boldsymbol{T}^{n}, 2\right)$ et $\boldsymbol{E}\left(\boldsymbol{T}^{n}, \mathbf{1}\right)$ est bien la variété de Picard de $T^{n}$. Il nous reste donc à montrer qu'il y a une correspondance biunivoque entre $\boldsymbol{N}\left(\boldsymbol{T}^{n}, 2\right)$ et $P^{n-1}$. Cette démonstration sera donnée au 6.2.

6. 2. Puisque $N(2, C) \cong C$, tout fibré dans $N\left(T^{n}, 2\right)$ est défini par une représentation $\phi$ du groupe fondamental $I I$ de $T^{n}$ de la forme

$$
\phi(a)=\left(\begin{array}{cc}
1 & \phi^{\prime}(a) \\
0 & 1
\end{array}\right), \quad a \in \Pi .
$$

(cf. la proposition 3.4 et la définition de $N\left(T^{n}, m\right)$ ) 
Soit $T^{n}=A / \Pi, A$ étant l'espace vectoriel complexe de dimension $n$ et $\Pi$ étant un sous-groupe discret de rang $2 n$ qui est identifié avec le groupe fondamental de $T^{n}$. Soit $\phi$ une représentation de $I$ de la forme (1). Alors $\phi^{\prime}$ est une représentation additive de $\Pi$ dans $C: \phi^{\prime}(a+b)=\phi^{\prime}(a)+\phi^{\prime}(b)$, $a, b \in \Pi$. Nous appelons une représentation de la forme (1) une représentation unipotente de $I I$ et la representation additive $\phi^{\prime}$ le représentation additive de II associée à $\phi$.

Lемме 6.1. Si le fibré defini par une représentation unipotente $\phi$ de $\Pi$ est décomposable, il est trivial.

Soit $E$ le fibré vectoriel défini par la représentation unipotente $\phi$ et supposons que $E$ soit décomposable: $E=E_{1} \oplus E_{2}, E_{i}$ étant des fibrés de fibre $C$. Puisque $\phi$ est unipotente, on a $\operatorname{det}(E)=I, I$ désignant le fibré vectoriel trivial de fibre $C$ (pour la notion de déterminant d'un fibré, voir Hirzebruch [4]). D'autre part, on a $\operatorname{det}\left(E_{1} \oplus E_{2}\right)=E_{1} \otimes E_{2}$. Par suite, on a $E_{1} \otimes E_{2}=I$ et donc $E_{2}=E_{1}^{*}, E_{1}^{*}$ désignant le dual de $E_{1}$. D'autre part $\phi$ étant unipotente, le fibré $E$ possède une section holomorphe $s \neq 0$. Soient $p_{1}$ et $p_{2}$ les projections canoniques de $E=E_{1} \oplus E_{2}$ sur $E_{1}$ et sur $E_{2}$ respectivement. Alors $p_{i} \circ s$ est une section holomorphe de $E_{i}$ et il n'est pas possible que $p_{1} \circ s=p_{2} \circ s=0$, car $s \neq 0$. Donc un des $E_{i}$, soit $E_{1}$, possède une section holomorphe non nulle. Alors d'après la proposition $3.6, E_{1}=I$. On a alors $E_{2}=I^{*}=I$ et par suite $E=I \oplus I$. $E$ est donc trivial.

Nous désignons dans tout ce qui suit par $E_{\beta}$ le fibré vectoriel défini par la représentation unipotente $\phi$ de $\Pi$. Le lemme suivant est facile à démontrer.

Lemme 6.2. Soit $\phi$ et $\psi$ des représentations unipotentes de $I 1$. Pour que $E_{\varnothing} \cong E_{\psi}$ il faut et il suffit qu'il existe une application holomorphe $f$ de $A$ dans $G L(2, C)$ telle que $f(x+a)=\psi(-a) \cdot f(x) \cdot \phi(a)$ pour tout $x \in A$ et $a \in \Pi$.

L'espace vectoriel complexe $A$ peut être considéré comme un espace vectoriel réel que l'on désigne par $A_{0}$. Désignons par $A_{0}^{* c}$ l'espace vectoriel complexe des formes linéaires à valeurs complexes sur $A_{0}$. On peut considérer le dual $A^{*}$ de $A$ comme un sous-espace complexe de $A_{0}^{* c}$. Soit maintenant $\phi^{\prime}$ une représentation additive de $\Pi$ dans $C$. Puisque $A_{0}$ est engendré sur $R$ par $\Pi$, $\phi^{\prime}$ se prolonge à une forme linéaire à valeurs 'complexes sur $A_{0}$. Réciproquement, la restriction à $\Pi$ d'une formẹ linéaire à valeurs complexes sur $A_{0}$ 
est une représentation additive de $I$. La correspondance ainsi définie entre l'ensemble des représentations additives de $I$ et $A_{0}^{* c}$ est biunivoque, car $A_{0}$ est engendré par $\Pi$. On identifie donc une représentation additive de $\Pi$ avec un élément de $A_{0}^{* c^{c}}$.

Lemme 6.3. Soit $\phi$ une représentation unipotente de II. Pour que le fibré $E_{\phi}$ soit décomposable, il faut et il suffit que $\phi^{\prime} \in A^{*}$, où $\phi^{\prime}$ désigne la représentation additive de $I I$ associée à $\phi$.

Supposons d'abord que $E_{\phi}$ soit décomposable. $E_{\phi}$ est alors trivial d'après le lemme 6.1. Il existe alors une application holomorphe $f$ de $A$ dans $G L(2, C)$ telle que $f(x+a)=f(x) \cdot \phi(a)$ pour tout $a \in \Pi$ et $x \in A$ (cf. Lemme 6.2). Remplacant $f(x)$ par $f(0)^{-1} \cdot f(x)$ au besoin, on peut supposer que $f(0)=I$. Posons

$$
f(x)=\left(\begin{array}{cc}
u(x) & v(x) \\
w(x) & z(x)
\end{array}\right)
$$

Par un calcul facile on déduit de l'équation $f(x+a)=f(x) \cdot \phi(a)$ les équations suivantes: $u(x+a)=u(x), w(x+a)=w(x), v(x+a)=\phi^{\prime}(a) \cdot u(x)+v(x)$, $z(x+a)=\phi^{\prime}(a) \cdot w(x)+z(x)$ pour tout $a \in I$ et $x \in A$. Puisque $u$ et $w$ sont des fonctions holomorphes, on en déduit que $u$ et $w$ sont constantes. De plus, puisque $f(0)=I$, on a $u(0)=1, w(0)=0$. On a donc $u \equiv 1$ et $w \equiv 0$. Alors la dernière équation se réduit à $z(x+a)=z(x)$. Par un raisonnement analogue on voit que $z \equiv 1$. La troisième équation se réduit donc à $v(x+a)=\phi^{\prime}(a)+v(x)$. Alors la différentielle $d v$ est invariante par les translations par les éléments de $I I$. Il existe alors une l-forme holomorphe $\omega$ sur $T^{n}$ telle que $p^{*} \cdot \omega=d v, p$ désignant la projection canonique de $A$ sur $T^{n}$. Soit $L_{w}$ la forme linéaire sur $A$ définie par $\omega$ (voir 2.C). Alors $L_{w}(a)=\int_{0}^{a} p^{*} \omega=\int_{0}^{a} d v=\phi^{\prime}(a)$ pour tout $a \in \Pi$ et par suite $\phi^{\prime} \in A^{*}$. Soit, réciproquement, $\phi^{\prime} \in A^{*}$. Toute forme linéaire sur $A$ est de la forme $L_{w}, \omega$ étant une l-forme holomorphe sur $T^{n}$ (voir, 2. $C$ ). Il existe donc une l-forme holomorphe $\omega$ sur $T^{n}$ telle que $\phi^{\prime}(a)=\int_{0}^{a} p^{*} \cdot \omega$ pour tout $a \in \Pi$. Soit $v(x)=\int_{0}^{x} p^{*} \cdot \omega$. On a alors $v(x+a)=\phi^{\prime}(a)+v(x)$ pour tout $x \in A$ et $a \in \Pi$. Soit

$$
f(x)=\left(\begin{array}{cc}
1 & v(x) \\
0 & 1
\end{array}\right) .
$$


Alors $f(x)$ est une application holomorphe de $A$ dans $G L(2, C)$ et on a $f(x+a)$ $=f(x) \cdot \phi(a)$ pour tout $x \in A$ et $a \in I I$. Il résulte du lemme 6.2 que $E_{s}$ est trivial. Le lemme 6.3 est ainsi démontré.

Lemme 6.4. Soient $\phi$ et $\phi$ des représentations unipotentes de $I 1$ telles que $E_{\zeta}$ et $E_{\psi}$ soient indécomposables. Pour que $E_{\sigma} \cong E_{\Downarrow}$ il faut et il suffit qu'il existe un nombre complexe $c \neq 0$ tel que $c \cdot \phi^{\prime} \equiv \psi^{\prime}\left(\bmod A^{*}\right)$, où $\phi^{\prime}$ et $\psi^{\prime}$ désignent les représentations additives associées à $\phi$ et $\dot{\psi}$ respectivement.

Supposons d'abord que $E_{\varsigma} \cong E_{\psi}$. D'après le lemme 6.2 il existe alors une application holomorphe $f$ de $A$ dans $G L(2, C)$ telle que $f(x+a)=\psi(-a) \cdot f(x)$ - $\phi(a)$ pour tout $x \in A$ et $a \in \Pi$. Soit

$$
f(x)=\left(\begin{array}{cc}
u(x) & v(x) \\
w(x) & z(x)
\end{array}\right)
$$

Par un calcul facile, on déduit de l'équation $f(x+a)=\phi(-a) \cdot f(x) \cdot \phi(a)$ les équations suivantes.

a) $u(x+a)=u(x)-\psi^{\prime}(a) \cdot w(x)$

b) $w(x+a)=w(x)$,

c) $z(x+a)=z(x)+\phi^{\prime}(a) \cdot w(x)$,

d) $v(x+a)=v(x)+\phi^{\prime}(a) \cdot u(x)-\phi^{\prime}(a) \cdot \psi^{\prime}(a) \cdot w(x)-\phi^{\prime}(a) \cdot z(x)$.

Il résulte de $\mathrm{b}$ ) que $w=$ const., $w \equiv w_{0}$. Supposons que $w_{0} \neq 0$. Posons $u^{\prime}(x)$ $=-\frac{1}{w_{0}} \cdot u(x)$. On a alors $u^{\prime}(x+a)=u^{\prime}(x)+\psi^{\prime}(a)$. L'existence d'une telle fonction holomorphe $u^{\prime}$ implique que $E_{\psi}$ est décomposable (cf. la démonstration du lemme 6.3). Cette contradiction montre que $w_{0}=0$. Il résulte alors des équations a) et $\mathrm{b})$ que les fonctions $u$ et $z$ sont constantes. Soit $u \equiv u_{0}$ et $z \equiv z_{0}$. Alors det $f(x)=u_{0} \cdot z_{0} \neq 0$, par suite $u_{0} \neq 0$ et $z_{0} \neq 0$. L'équation d) s'écrit $v(x+a)=v(x)+u_{0} \cdot \phi^{\prime}(a)-z_{0} \cdot \psi^{\prime}(a)$. Le différentielle $d v$ est invariante par les translations par les éléments de $\Pi$ et par suite il existe une l-forme holomorphe $\omega$ sur $T^{n}$ telle que $d v=p^{*} \omega$. On a alors $L_{w}(a)=u_{0} \cdot \phi^{\prime}(a)-z_{0} \cdot \psi^{\prime}(a)$ pour tout $a \in I$ (cf. la démonstration du lemme 6.3). Soit $c=u_{0} / z_{0}$. Alors $c \cdot \phi^{\prime}-\psi^{\prime}=1 \frac{1}{z_{0}} \cdot L_{w} \in A^{*}$.

Supposons, réciproquement, qu'il existe un nombre complexe $c \neq 0$ tel que $c \cdot \phi^{\prime}-\psi^{\prime} \in A^{*}$. Il existe alors une fonction 'holomorphe $v$ sur $A$ telle que $v(x+a)=v(x)+c \cdot \phi^{\prime}(a)-\psi^{\prime}(a)$ pour tout $x \in A$ et $a \in \Pi$. Soit 


$$
f(x)=\left(\begin{array}{cc}
c & v(x) \\
0 & 1
\end{array}\right) .
$$

Alors $f$ est une application holomorphe de $A$ dans $G L(2, C)$ et on a $f(x+a)$ $=\psi(-a) \cdot f(x) \cdot \psi(a)$ pour tout $x \in A$ et $a \in I I$. Alors $E_{\S} \cong E_{\psi}$ par le lemme 6.2. Le lemme 6.1 est donc établi.

Nous allons maintenant démontrer qu'il y a une correspondance biunivoque entre $N\left(\boldsymbol{T}^{n}, 2\right)$ et $P^{n-1}$. Soit $N$ l'ensemble des fibrés vectoriels indécomposables

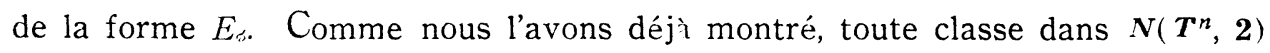
peut être représentée par un fibré clans $N$. Réciproquement toute classe de fibrés représentée par un fibré dans $N$ appartient à $N\left(T^{n}, 2\right)$. Si $E_{\not \phi} \in N$, on a $\phi^{\prime} \notin A^{*}$, car $E_{\S}$ est indécomposable. Soit maintenant $L=A_{\uparrow}^{* c} / A^{*}$. L est un espace vectoriel complexe de dimension $n$. Soit $\theta$ l'application de $N$ sur $L-\{0\}$ définie par $\theta\left(E_{t}\right)=q\left(\phi^{\prime}\right), q$ désignant la projection canonique de $A_{*}^{*}$ sur $L$ (L'application $\theta$ est surjectif, car pour toute représentation additive $\phi^{\prime}$ de $I I$ dans $C$, il existe une représentation unipotente de $\phi$ telle que la représentation additive associée soit égale à $\phi^{\prime}$ donnée). D'après le lemme 6.4 , on a $E_{5} \simeq E_{\text {c }}$ si et seulement si $\theta\left(E_{\xi}\right)$ et $\theta\left(E_{\psi}\right)$ sont collinéaires. L'application $\theta$ définit donc une application biunivoque de $\boldsymbol{N}\left(\boldsymbol{T}^{n}, 2\right)$ sur $P^{n-1}$. Le théorème $\bar{j}$ est ainsi démontré.

\section{BiBLIOGRAPHIE}

[1] M. F. Atiyah, Complex analytic connections in fibre bundles, Trans. Amer. Math. Soc., vol. 85 (1957), pp. 181-207.

[2] M. F. Atiyah, Vector bundles over an elliptic curve, Proc. London Math. Soc., vol. 7 (1957), pp. $414-452$

[3] M. F. Atiyah, On the Krull-Schmidt theorem with application to sheaves, Bull. Soc. Math. de France, Tome 84 (1956), pp. 307-317.

[4] A. Grothendieck, Sur la classification des fibrés holomorphes sur la sphère de Riemann, Amer. Jour. Math., vol. 79 (19.57), pp. 121-138.

[う] F. Hirzebruch, Neue topologische Methoden in der algebraischen Geometrie, Erg. der Math., Springer (1956).

[6] K. Kodaira and D. Spencer, Groups of complex line bundles over compact Kähler varieties, Proc. Nat. Acad. Sci., vol. 39 (1953), pp. 868-872.

[7] A. Morimoto, Sur le groupe d'automorphismes d'un espace fibré principal analytique complexe, Nagoya Math. J. vol. 13 (1958), pp. 157-168.

[8] S. Murakami, à paraitre.

[9] K. Nomizu, Lie groups and differential geometry, Publication of Math. Soc. of Japan, $2(19,5)$. 
[10] H. C. Wang, On invariant connections over a principal fibre bundle, Nagoya Math. J. vol. 13 (1958), pp. 1-19.

[11] A. Weil, On Picard varieties, Amer. Journ. Math., vol. 74 (1952), pp. 865-894.

Institut de Mathématiques,

Université de Nagoya 